\title{
An On-Line Harmonic Elimination Pulse Width Modulation Scheme for Voltage Source Inverter
}

\author{
Zainal Salam ${ }^{\dagger}$ \\ ${ }^{\dagger}$ Faculty of electrical Eng., Universiti Teknologi Malaysia, Skudai, Johor, Malaysia
}

\begin{abstract}
This paper proposes a new harmonic elimination PWM (HEPWM) scheme for voltage source inverters (VSI) based on the curve fittings of certain polynomials functions. The resulting equations to calculate the switching angle of the HEPWM require only the addition and multiplication processes; therefore any number of harmonics to be eliminated and the fundamental amplitude of the pole switching waveform $(N P 1)$ can be controlled on-line. An extensive angle error analysis is carried out to determine the accuracy of the algorithm in comparison to the exact solution. To verify the workability of the technique, an experimental single phase VSI is constructed. The algorithm is implemented on a VSI using a 16-bit microprocessor. The results obtained from the test rig are compared to the theoretical prediction and the results of the MATLAB simulations.
\end{abstract}

Key Words: Elimination, Harmonics, Inverter, PWM, Switching loss

\section{INTRODUCTION}

The advantages of harmonic elimination pulse width modulation (HEPWM) over the conventional sinusoidal PWM (SPWM) for Voltage Source Inverters (VSI) are widely recognized. These include [1], [2]:

- For a given VSI switching frequency, the incidence of the first nonzero harmonic is almost double compared to the SPWM scheme, resulting in a superior pole switching waveform harmonic spectrum.

- About a 50\% reduction in the inverter switching frequency is achieved which contributes to a reduction in the switching losses of the VSI.

- A higher pole switching waveform fundamental amplitude is attainable before the minimum pulse-width limit is reached

- Over-modulation is possible, contributing to higher utilization of the power conversion process.

Considering these issues, HEPWM can be a useful alternative to the more popular SPWM, especially for the high power inverters used in the mains and drives applications. Under these circumstances, switching losses are a major concern and HEPWM provides an ideal solution, especially for inverters that are switched in the low $\mathrm{kHz}$ range. However, despite its benefits, the widespread use of HEPWM is somewhat hindered because the equations to calculate the switching angles are non-linear and transcendental; restricting it to offline computation using extensive numerical techniques such as the Newton-Raphson method [3]. The problem with this

\footnotetext{
Manuscript received Jul. 30, 2009; revised Dec. 1, 2009

† Corresponding Author: zainals@ fke.utm.my

Tel: +607-5536187, Fax: +067-5566272, Universiti Teknologi Malaysia

Faculty of electrical Eng., Universiti Teknologi Malaysia, Malaysia
}

method is that if the initial values are not chosen correctly, it may result in large iteration cycles which can take many hours to complete. In some cases the solution can not converge at all.

Assuming that all the HEPWM angles are successfully calculated using an off-line computer, they are then stored in memory and called upon whenever the PWM waveform is to be constructed. This approach is popularly known as Programmed HEPWM and it is well documented by [4]. Although simple, it requires a large amount of memory to store all the calculated angles. This is particularly troublesome when frequent changes in the modulation parameters, such as the amplitude or frequency ratios, are needed. For motor drive applications where the operating frequency varies over a wide range, interpolations in-between points may be required for greater accuracy, thus adding complexity to the algorithm. However for an inverter that has a fixed frequency output, e.g. for utility applications, the use of programmed HEPWM is acceptable as recently demonstrated by [5], [6] .

Research has been carried out to derive HEPWM equations for on-line calculation. The main advantage of the on-line approach is the absence of memory to store the switching angles. Furthermore all the angles can be calculated as demanded by the modulation parameters. It appears that all of the schemes in this category require some sort of approximation of the "exact" HEPWM switching angles to arrive at simpler equations suitable for real time computation. One of the early attempts for such work was carried out by [7]. He derived a set of non-transcendental equations for a near-optimal solution using sine-wave approximations. Using this scheme, the HEPWM switching angle equations are reduced to a non-transcendental form that permits on-line computation. Another HEPWM 
scheme, based on regular sampled PWM was suggested by [8]. The same author also carried out similar work for a space vector modulation scheme [9]. A HEPWM scheme using bipolar switching was proposed in [10]. Other, more complex, methods were proposed by Liang using the Walsh functions [11] and by Kato using a homotopy-based computation [12]. Chiasson later proposed a conversion of the HEPWM equation to a set of polynomials and by applying the resultant theory derived a complete solution for the switching angles [13]. This technique has been applied to multilevel inverter structures [14], and further extended to multilevel inverters with nonequal DC sources [15]. More recently a HEPWM scheme for multilevel inverter using genetic algorithm was proposed [16].

On-line HEPWM schemes are normally implemented using high performance digital signal processors. This is inevitable due to the significant computing power required for the switching angles calculations. However, it would be desirable if a general microprocessor could be used with the condition that a reasonably accurate output waveform could be achieved. However, to use such a microprocessor, the HEPWM algorithm must be efficient enough to ensure sufficient time to calculate the pulse width, thus avoiding the need for memory storage. For this reason, this paper proposes a simple on-line HEPWM scheme that can be suitably implemented using a fixed point microprocessor. It is based on the polynomial curve fitting of the trajectories of the exact HEPWM angles. It will be shown that the proposed scheme allows for efficient realtime computations with acceptable error margins. The viability of this scheme is validated using MATLAB simulations and hardware results.

\section{DeRIVATION OF ON-Line HEPWM EQUations}

A generalized bipolar PWM waveform with $M$ number of chops per half-cycle is depicted in Fig. 1. It is assumed that this waveform is periodic and has half-wave symmetry with per unit amplitude. Such a waveform is the representation for the pole switching waveform of a three phase inverter, i.e. voltage from one of the phases to the virtual ground. For the line to line voltage of the inverter output, the triplen (multiple of three) harmonics are cancelled. In Fig. 1, a basic square wave is chopped and a relationship between the number of chops and the number of possible harmonics that can be eliminated is derived. The odd switching angles $\alpha_{1}, \alpha_{3}, \alpha_{5} \ldots$ etc. define the falling edge transitions and the even switching angles $\alpha_{2}$, $\alpha_{4}, \alpha_{6} \ldots$ etc. define the rising edge transitions. Since the waveform is quarter-wave symmetric, only the odd harmonics exist (i.e. $B_{n}=0$ ) and they are given by the following Fourier Series representation:

$A_{n}=\frac{4}{n \pi}\left[1+2 \sum_{k=1}^{m}(-1)^{k} \cos n \alpha_{k}\right]$ odd harmonics only

Equation (1) has $m$ variables $\left(\alpha_{1}\right.$ to $\left.\alpha_{m}\right)$ and a set of solutions is obtainable by equating any $m-1$ harmonics to zero and assigning a value to the fundamental. Thus both the harmonic incidents and the fundamental components can be independently controlled. These equations are nonlinear as

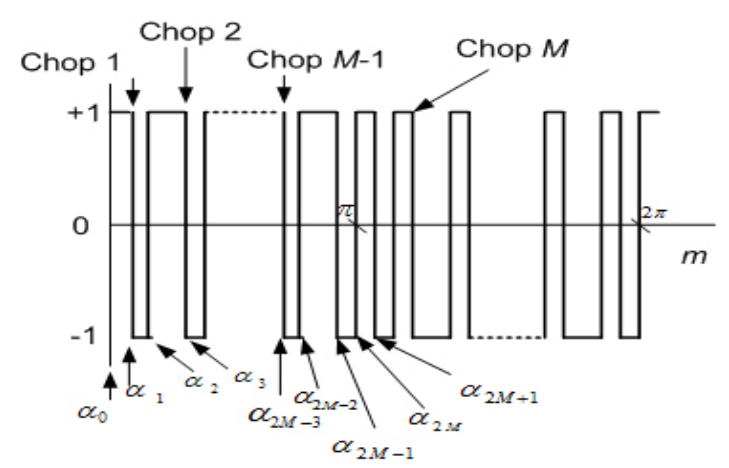

Fig. 1. Generalized quarter-wave symmetric HEPWM.

well as transcendental. A solution of these equations can be obtained using the Newton-Raphson iteration method:

$$
\begin{aligned}
& {\left[\begin{array}{r}
\alpha_{1} \\
\alpha_{2} \\
\vdots \\
\alpha_{m}
\end{array}\right]_{p+1}=\left[\begin{array}{c}
\alpha_{1} \\
\alpha_{2} \\
\vdots \\
\alpha_{m}
\end{array}\right]_{p}}
\end{aligned}
$$

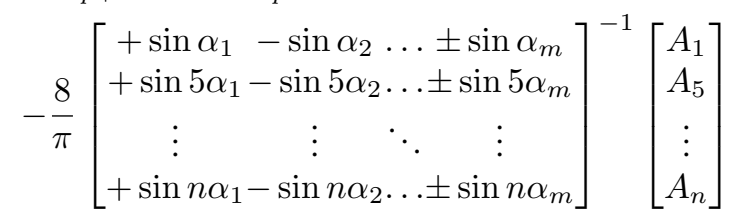

The trajectories for the switching angles $\left(\alpha_{1} \ldots \alpha_{m}\right)$ versus the per-unit amplitude of the fundamental component of the pole switching waveform (NP1) for an odd number of switching per quarter cycle can be obtained by MATLAB computation. Figs. 2(a) and (b) show examples of trajectories calculated for $m=3$ and 13, respectively. From Figs. 2(a) and (b) the highest intersection point for the trajectories with the $\mathrm{y}$-axis is $60^{\circ}$. Furthermore, the switching angle $\alpha_{m}$ is equal to $60^{\circ}$ when $N P 1$ equals zero for all values of $m$. From Fig. 3 the angular separation of the trajectories at the y-axis can be defined as:

$$
\text { Angular separation }=\frac{2 \times 60^{\circ}}{m+1}, \quad m \text { is odd }
$$

It can also be seen from Fig. 3 that for $N P 1$ ranges from 0 to 0.8 , the trajectories approximate a straight line. This region is denoted as the linear slope region. Hence a straight-line approximation of the trajectories could be used. However, for a $N P 1$ that is greater than 0.8 , the trajectories are no longer straight lines. This is defined as the non-linear slope region. Nevertheless, the straight-line approximation could still be used with an error correction scheme for the region of $N P 1$ that is greater than 0.8. Observing Figs. 2(a)-(d), the trajectories of the odd and the even switching angles are parallel lines over most of the range of $N P 1$. Also the slopes of the trajectories reduce with increasing values of $m$. Therefore, to obtain a relationship between the slopes of the trajectories for different values of $m$, the slopes should first be normalized towards the angular separation of the trajectories. At $N P 1=0.8$, the 


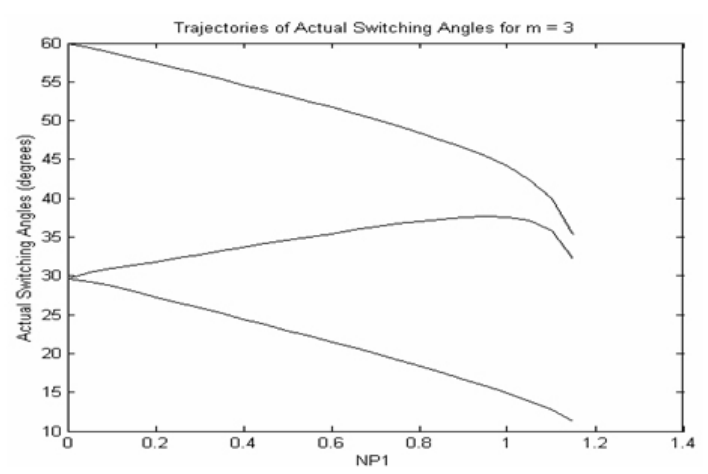

(a)

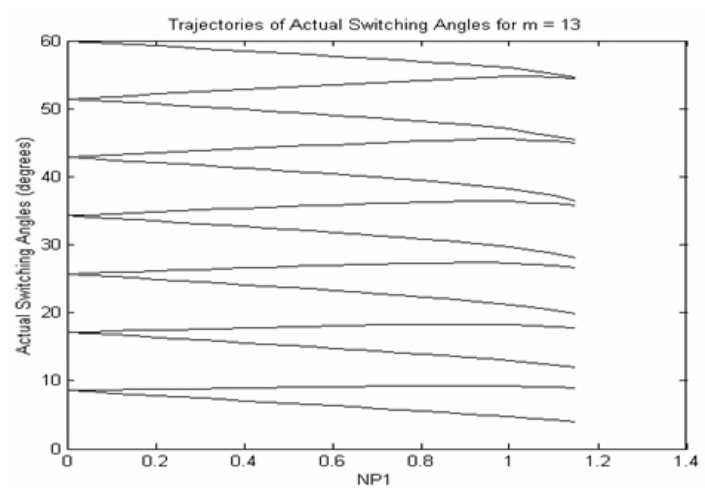

(b)

Fig. 2. Trajectories of switching angles for an odd number of switchings per quarter cycle. (a) $m=3$, (b)=13.

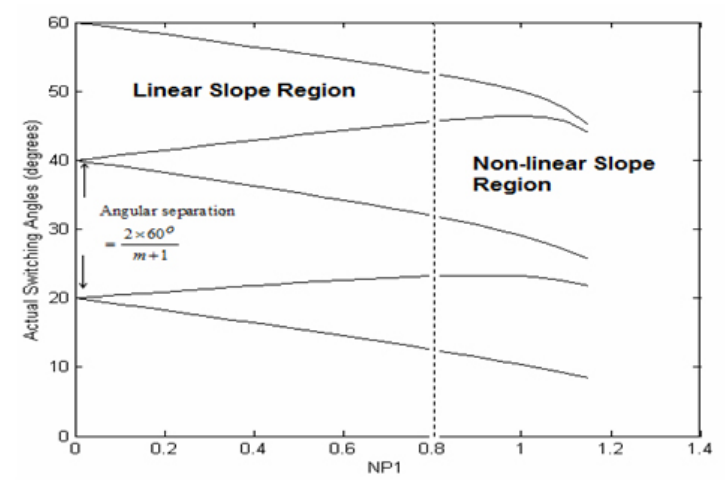

Fig. 3. Trajectories for $m=5$ to illustrate angular separation.

normalized slope, $\Delta_{k}$, can be computed as:

$$
\begin{aligned}
\Delta k & =\frac{\frac{60^{\circ}(k+1)}{(m+1)}-\alpha_{k}}{\frac{2 \times 60^{\circ}}{m+1}}, \text { for } k \text { odd } \\
\Delta k & =\frac{\alpha_{k}-\frac{60^{\circ}(k)}{(m+1)}}{\frac{2 \times 60^{\circ}}{m+1}}, \quad \text { for } k \text { even }
\end{aligned}
$$

Equations (4) and (5) are used to plot out $\Delta_{k}$ versus $k$ on separate graphs for odd and even values of $k$ as shown in Figs. 4 and 5, respectively. Fig. 4 shows the variation of $\Delta_{k}$, for odd and even values of $k$, for several values of $m$. The graphs suggest that for odd values of $k$, the function $\Delta_{k}$

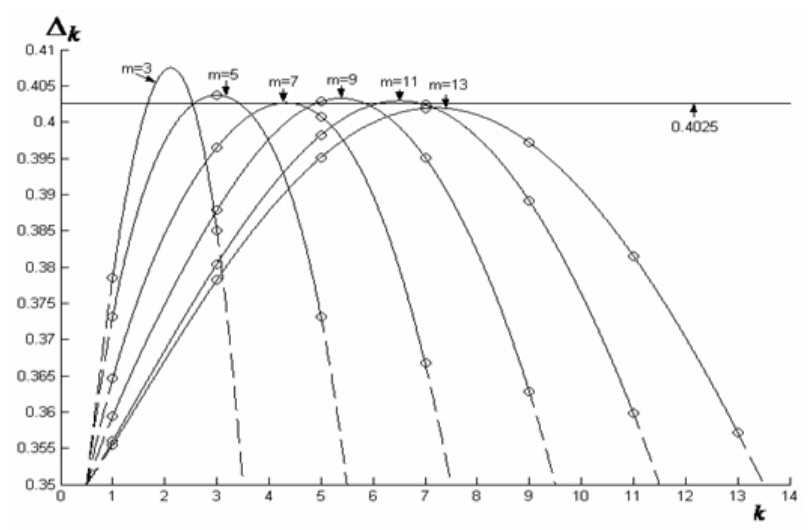

Fig. 4. $\Delta_{k}$ vs $k$ for several values of $m$ (for odd $k$ ).

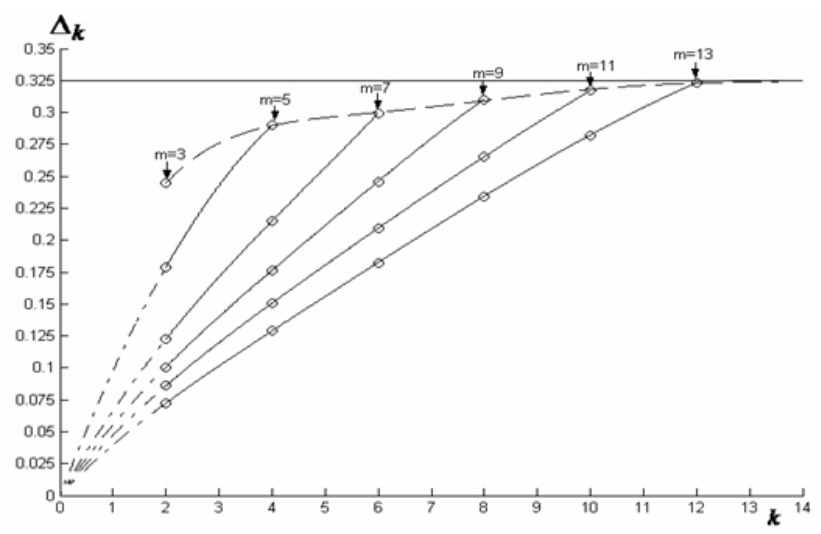

Fig. 5. $\Delta_{k}$ vs $k$ for several values of $m$ (for even $k$ ).

appears to be a polynomial (quadratic curves) with a nearly constant amplitude. Therefore, the obvious solution would be to apply a polynomial fit to the curves in Fig. 4. This leads to the equation of $\Delta_{k}$ for the odd values of $k$ :

$$
\Delta_{k}=-\frac{0.21}{m^{2}}\left(k-\frac{m+1}{2}\right)^{2}+0.4025, k \text { odd }
$$

The generalized equation for the odd switching angles, for any values of $m$ and $N P 1$, is given by:

$$
\alpha_{k}=\frac{60^{\circ}(k+1)}{m+1}-\left[\frac{2 \times 60^{\circ}}{m+1} \times \frac{\Delta_{k} \times N P 1}{0.8}\right], k \text { odd }
$$

Fig. 5 shows the variation of $\Delta_{k}$ for the even switching angles. Extrapolation shows that all the curves pass through the origin. For increasing values of $m$, the values of $\Delta_{k}$ appear to be asymptotic to a line drawn parallel to the $\mathrm{x}$-axis and intersecting the $\mathrm{y}$-axis at 0.325 . Taking this into account, the expression of $\Delta_{k}$ for the even values of $k$ will be in the form of:

$\Delta_{k}=-\frac{0.082}{(m-1)^{2}}[k-2.482(m-1)]^{2}+0.505-\frac{k}{m^{3}}$

The generalized algorithm for the even switching angles for any value of $m$ and $N P 1$, is then given by:

$\alpha_{k}=\frac{60^{\circ} \times k}{m+1}+\left[\frac{2 \times 60^{\circ}}{m+1} \times \frac{\Delta_{k} \times N P 1}{0.8}\right]$, for even $k$ 


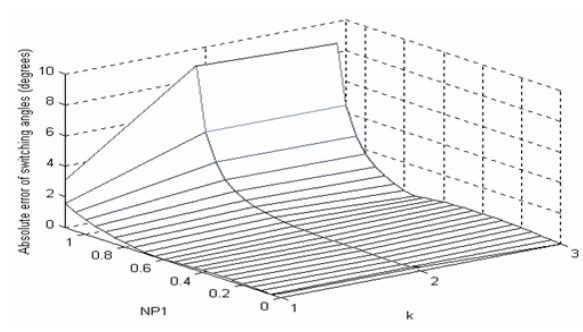

(a)

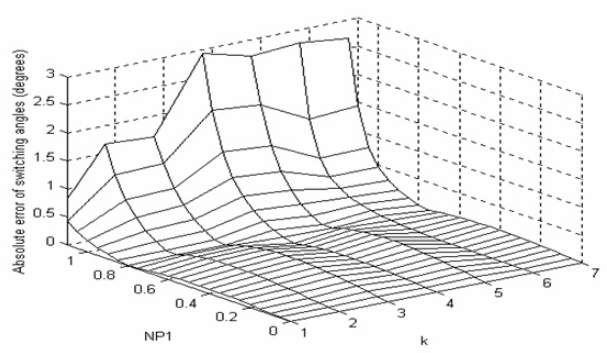

(b)

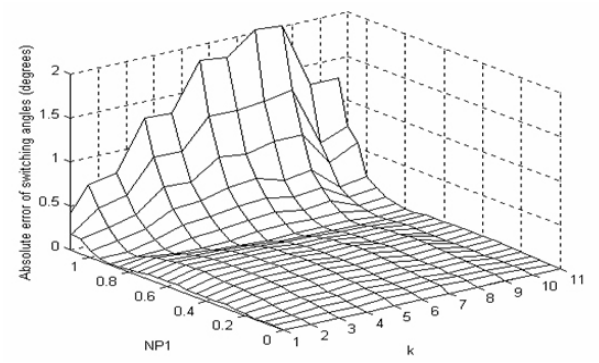

(c)

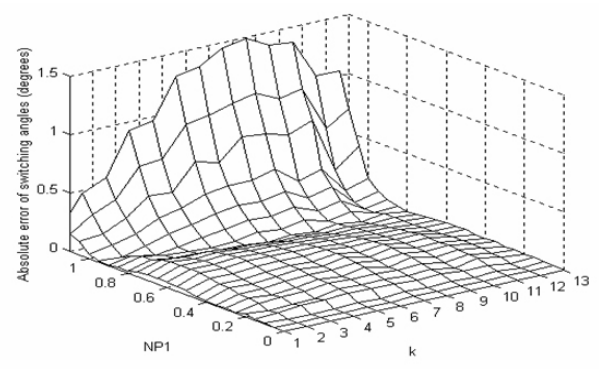

(d)

Fig. 6. Variation of switching angle errors for (a) $m=3$, (b) $m=7$, (c) $m=11$, (d) $m=13$.

Equations (6), (7), (8) and (9) can now be used to calculate the approximate switching angles for any values of $m$ and $N P 1$. These simple equations can then be implemented easily on any microprocessor which has the multiplication command in its instruction set. The simplicity of the algorithm allows for very fast and efficient generation of the HEPWM waveform.

\section{ANALYSIS OF THE ACCURACY OF THE ALGORITHM}

\section{A. Without error correction}

The accuracy of the derived equations is evaluated by calculating the difference between the approximate switching angles from the proposed method and the exact switching angles from the trajectories. The absolute difference is termed the angle error. The plots for the error angles versus $N P 1$ for

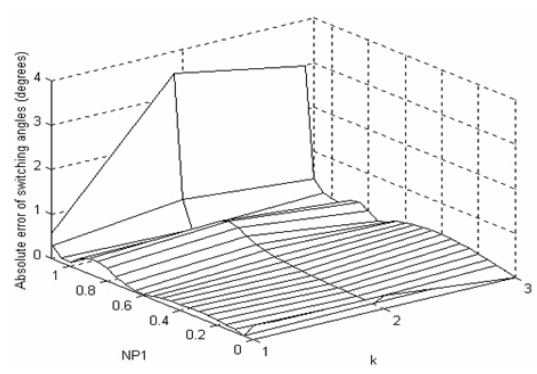

(a)

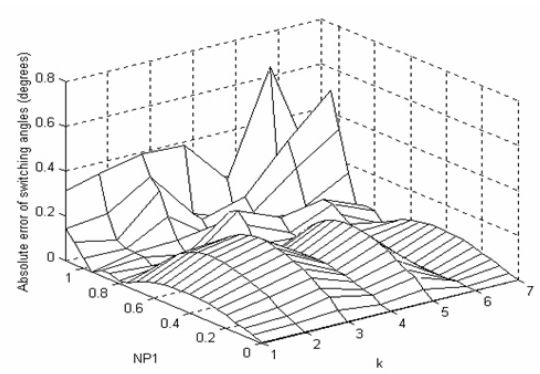

(b)

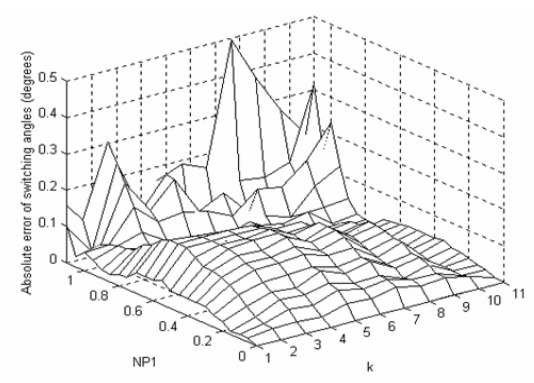

(c)

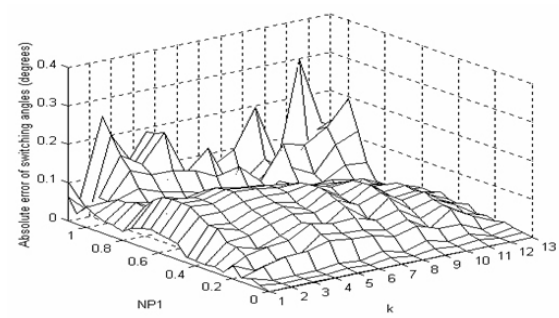

(d)

Fig. 7. Variation of error incorporating error correction for $N P 1>0.8$. (a) $m=3$, (b) $m=7$, (c) $m=11$, (d) $m=13$.

different values of $m$ are shown in Fig 6(a)-(d). For a $N P 1$ $<0.8$, the maximum angle error for every value of $m$ is very small. In addition the errors decrease for increasing values of $m$. This implies that the accuracy of the elimination technique improves with higher value of $m$. However for values of $N P 1$ that are greater than 0.8 , the errors increase drastically. The reason for this increase can be attributed to the departure of the trajectories from being straight lines for values of $N P 1$ above 0.8, as can be seen in Figs 2(a) and (b). The maximum angle errors for odd and even switching angles for $0<N P 1$ $\leq 0.8$ and $0.8<N P 1 \leq 1.15$ are tabulated in Tables 1 and 2 , respectively. 
TABLE I

MAXIMUM ANGLE ERRORS FOR $0<N P 1 \leq 0.8$

\begin{tabular}{|c|c|c|}
\hline$m$ & Max error odd $k$ (deg.) & Max error even $k$ (deg.) \\
\hline 3 & 0.6795 & 0.8967 \\
5 & 0.3242 & 0.4535 \\
7 & 0.2759 & 0.3469 \\
9 & 0.2136 & 0.2232 \\
11 & 0.1784 & 0.1582 \\
13 & 0.1533 & 0.1154 \\
\hline
\end{tabular}

TABLE II

MAXIMUM ANGLE ERRORS FOR $0.8<N P 1 \leq 1.15$

\begin{tabular}{|c|c|c|}
\hline$m$ & Max error odd $k$ (deg.) & Max error even $k($ deg.) \\
\hline 3 & 8.3785 & 8.6192 \\
5 & 4.2015 & 4.3793 \\
7 & 2.6184 & 2.8355 \\
9 & 2.2420 & 2.1003 \\
11 & 1.9446 & 1.9688 \\
13 & 1.4446 & 1.4038 \\
\hline
\end{tabular}

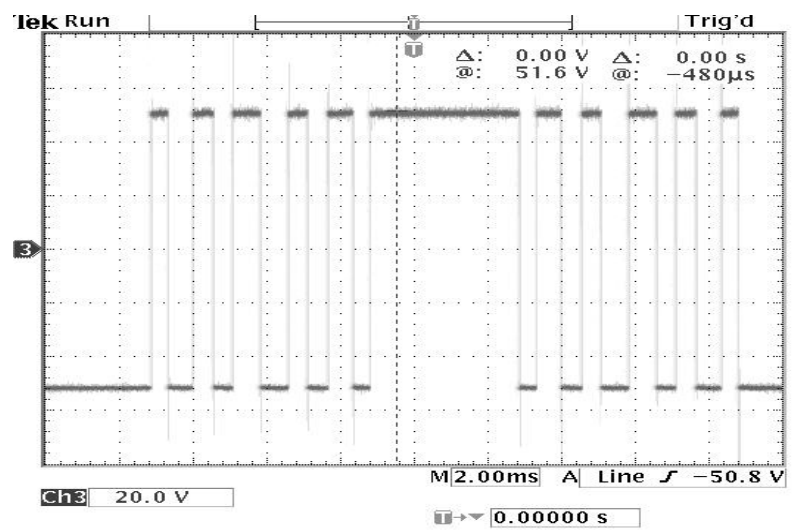

(a)

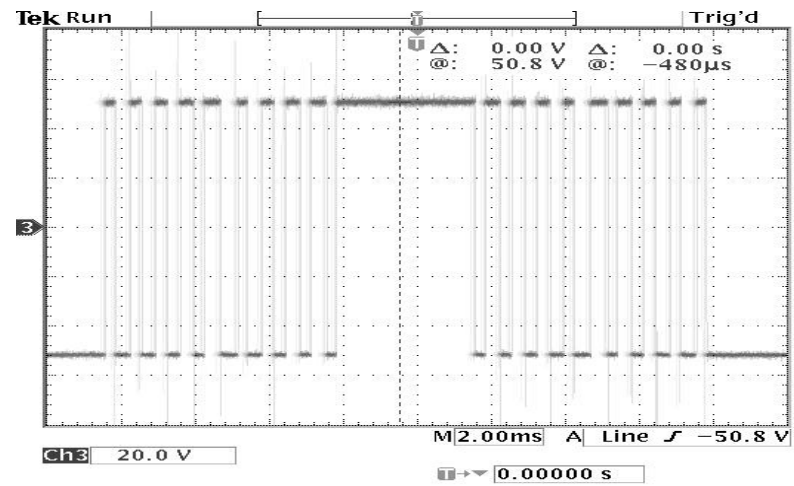

(b)

Fig. 8. Inverter output waveform an $N P 1=0.7$, fundamental frequency $=50 \mathrm{~Hz}$, (a) $m=5$ (b) $m-=9$.

\section{B. With Error Correction}

To account for the relatively large errors for a $N P 1>0.8$, an error correction factor is incorporated. For odd values of $k$ such that:

$$
\alpha_{k(\text { corrected })}=\alpha_{k}-\Delta D_{k}
$$

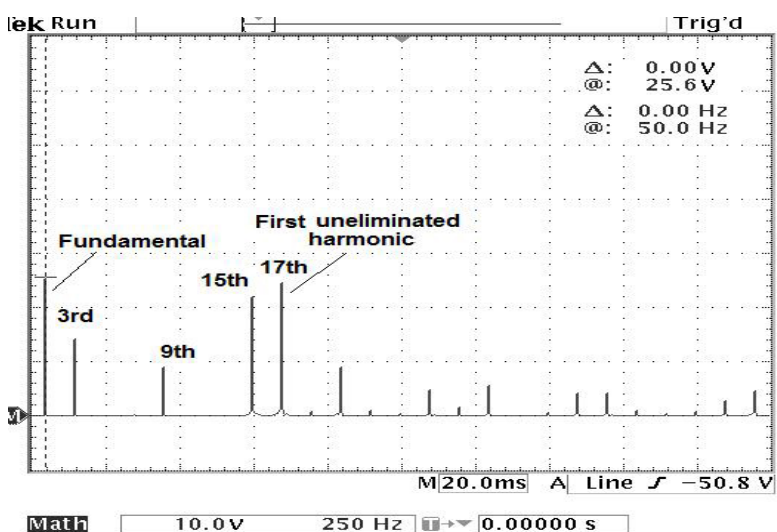

(a)

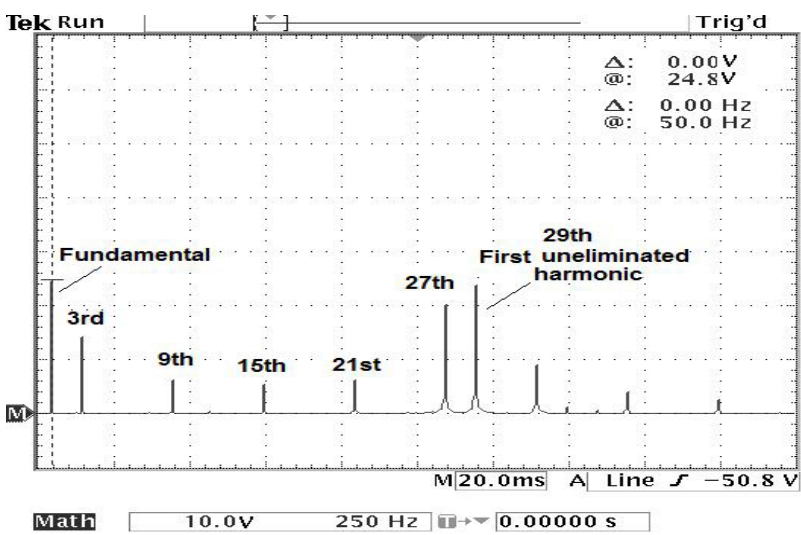

(b)

Fig. 9. Spectra of inverter output at $N P 1=0.7$, fundamental frequency $=$ $50 \mathrm{~Hz}$ (a) $m=5$ (b) $m=9$. Scale: Amplitude ( $y$-axis): $10 \mathrm{~V} /$ div. Frequency ( $x$-axis): $250 \mathrm{~Hz} /$ div. All triplens become zero when line to line voltage of a three phase is considered.

this is done with:

$$
\Delta D_{k}=\frac{(N P 1-0.8)^{2}}{0.09} \times\left[-\frac{52}{m}\left[\frac{k}{m+5}-0.5\right]^{2}+\frac{13}{m}\right],
$$

For even switching angles such that:

$$
\alpha_{k(\text { corrected })}=\alpha_{k}-\Delta D_{k}
$$

this is done with:

$$
\Delta D_{k}=\frac{(N P 1-0.8)^{2}}{0.09} \times\left[-\frac{52}{m}\left[\frac{k}{m+3}-0.5\right]^{2}+\frac{13}{m}\right],
$$

Figs. 7(a)-(f) show the absolute errors between the exact switching angles incorporating the correction factors. Comparing these to Figs. 6(a)-(d), respectively, the maximum errors have been reduced by a factor of 3-6.

\section{HARDWARE AND MATLAB VERIFICATION}

To assess the effectiveness of the HEPWM algorithm, a 200Wsingle phase $\mathrm{H}$-bridge inverter is designed and constructed. The inverter is fed a $50 \mathrm{~V}$ DC input voltage. The HEPWM waveform generation was implemented using a lowcost, fixed-point 16-bit Siemens 80C167 microcontroller. For 


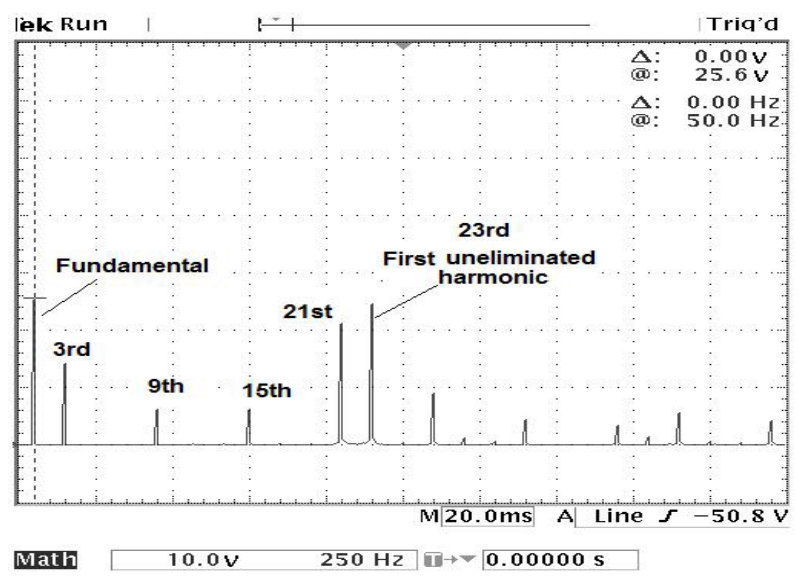

(a)

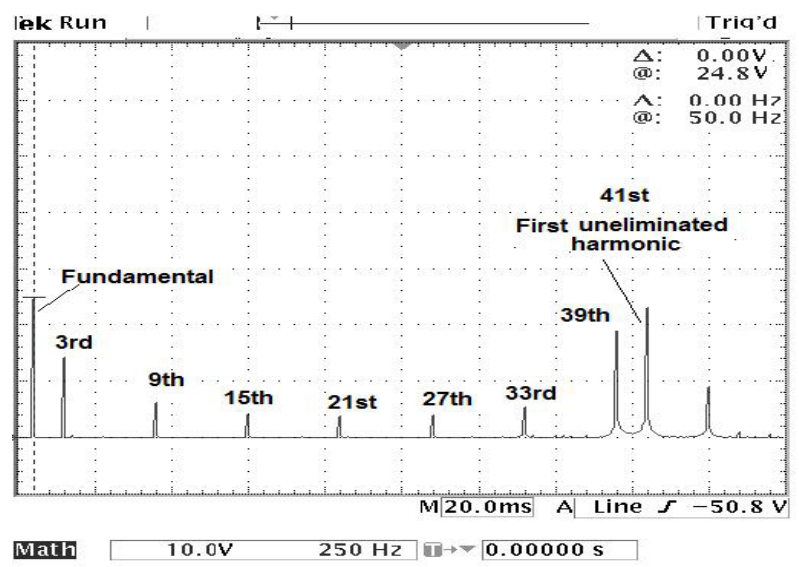

(b)

Fig. 10. Spectra of inverter output at $N P 1=0.7$, fundamental frequency $=$ $50 \mathrm{~Hz}$ (a) $m=7$ (b) $m=13$. Scale: Amplitude ( $y$-axis): 10V/div. Frequency ( $x$-axis): $250 \mathrm{~Hz} /$ div.

comparison, a MATLAB simulation of the same inverter system was carried out. If a three phase system is considered, its line to line harmonic incidence is the same as a single phase system, less the triplens. Therefore the triplens that exist in the spectra shown here are automatically cancelled when the line-to-line voltage is considered. Furthermore, it has to be noted that the inaccuracy due to the step size of the microcontroller sampling is not taken into account. Since the resolution of the PWM module in the microcontroller is $400 \mathrm{~ns}$, deviations from theoretical results are expected. In addition, the effects of the 1 us dead time introduced to each switching pulse are not considered. Clearly both factors affect the accuracy significantly, particularly at high values of $m$ where the HEPWM pulses are nearing their minimum widths.

\section{A. Cases for NP1 $<0.8$}

Figs. 8(a) and (b) show the output voltage waveform of the inverter at $N P 1=0.7$ for $m=5$ and $m=9$, respectively. For $m=5$, the expected eliminated harmonics (besides the triplens) are the 5th, 7th, 11th and 13th, while for $m=9$ the additional eliminated harmonics are the 17th, 19th, 23rd and 27th. Figs. 9(a) and (b) show the spectra plots of Figs. 8(a) and (b) computed using the FFT function of an oscilloscope. With

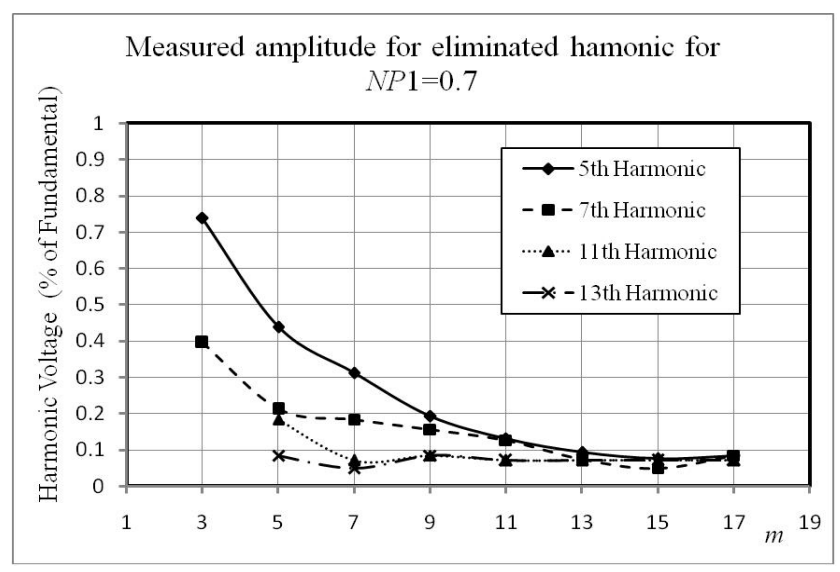

Fig. 11. Performance of the first four eliminated harmonics for different $m$ values at $N P 1=0.7$ measured at the inverter output.

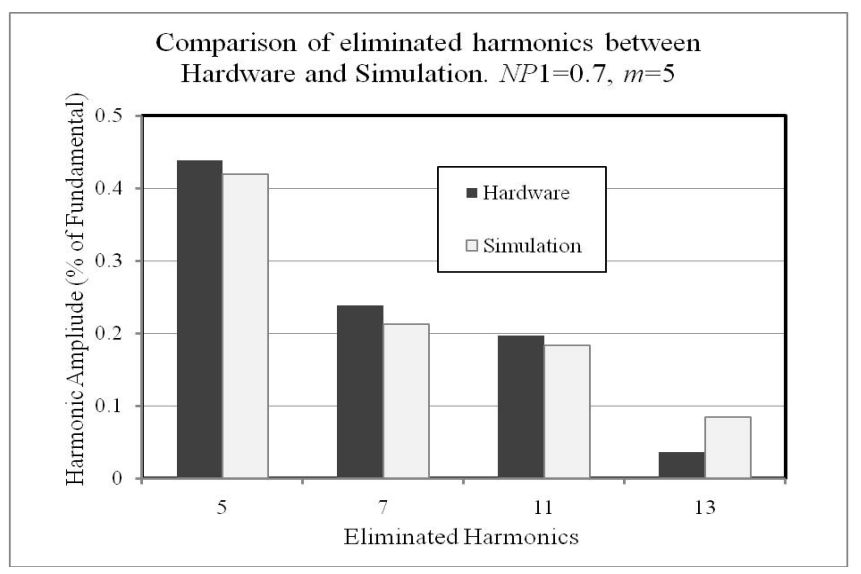

(a)

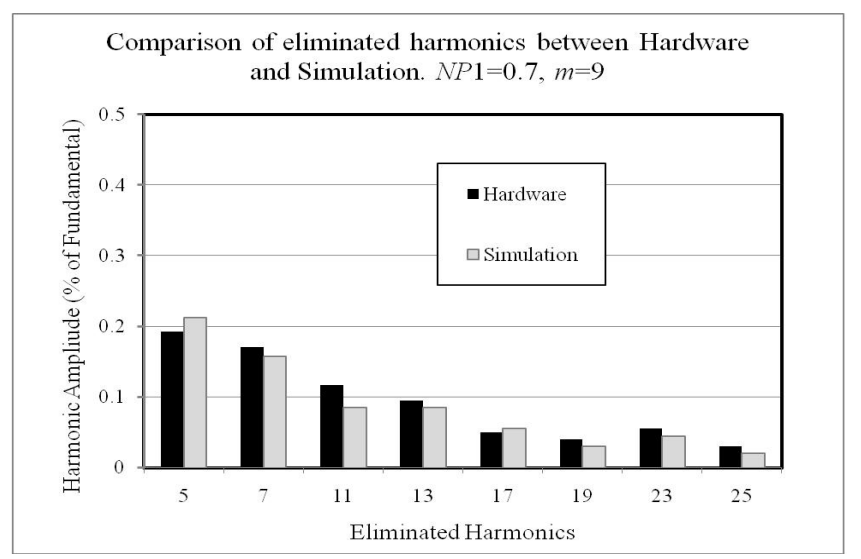

(b)

Fig. 12. Comparison of eliminated harmonics for inverter hardware and MATLAB simulation. (a) $m=5$, (b) $m=9$.

a frequency axis scaled at $250 \mathrm{~Hz} / \mathrm{div}$, it can be observed that all the specified harmonics are successful eliminated. For a three phase systems, since all triplens are absent, the first uneliminated harmonic is located at $(3 m+2)$. Thus if $m=5$, the first harmonic exists at the $17 \mathrm{th}$ or at $850 \mathrm{~Hz}$ for a $50 \mathrm{~Hz}$ fundamental frequency. For $m=9$, the first harmonic appears at the 31st. Figs. 10(a) and (b) show the spectral plots for other cases, e.g. $m=7$ and 13 , respectively.

Since the amplitudes of the eliminated harmonics are so 


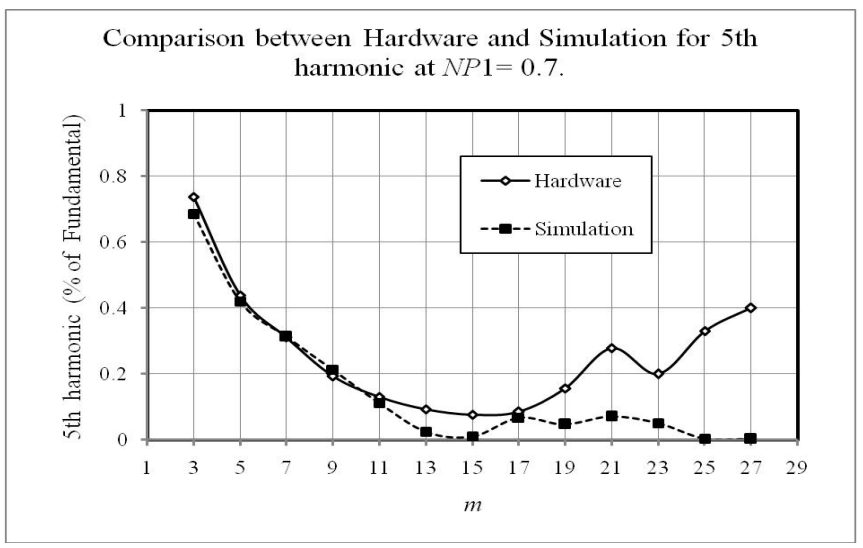

(a)

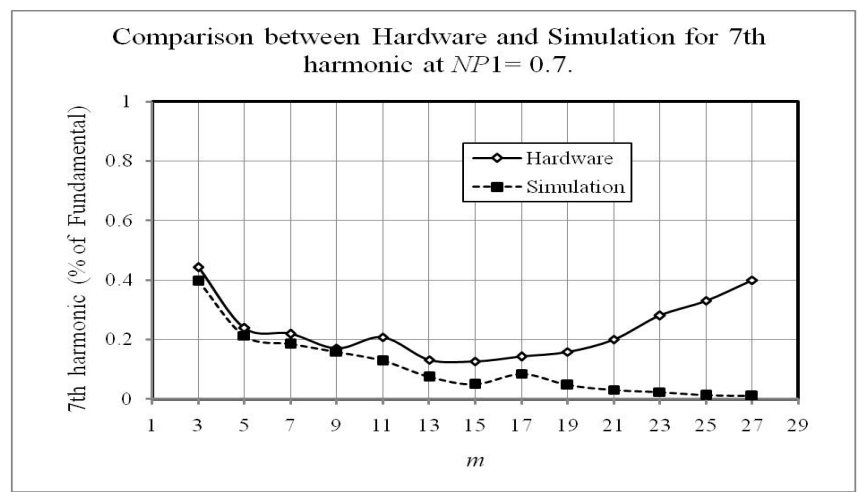

(b)

Fig. 13. Comparison of result from inverter hardware and MATLAB simulation for different valuse of $m$. (a) 5th harmonic (b) 7th harmonic.

small (compared to the fundamental), their presence on the spectral plots is not clearly visible. For clarity, the amplitudes of the first four eliminated harmonics for different values of $m$ are plotted in Fig. 11. As can be seen for $N P 1=0.7$, all the eliminated harmonic magnitudes are below $1 \%$ of the fundamental component. Furthermore, its accuracy improves as $m$ increases. This is consistent with the trend for the error angles observed in Figs. 6(a)-(d).

Figs. 12(a) and (b) compare the measured (from the hardware) and the simulated (MATLAB) eliminated harmonics for $m=5$ and $m=9$, respectively. Generally there is good agreement between the two approaches although for most cases, the performance of the measured harmonics is slightly inferior to the latter. The difference is due to the microcontroller resolution and the dead time of the practical inverter. To investigate further, measurements are made at higher values of $m$. For the 5th and 7th harmonics, as depicted in Figs. 13(a) and (b) respectively, as $m>17$ the accuracy deteriorates. This contradicts the trend observed for the error angles. However, the simulation plots on the same Figs appear to be consistent. This observation confirms the fact that for large value of $m$, the microprocessor resolution and the dead effects become increasingly important. In such cases they can not be ignored.

\section{B. Cases for NP1 $>0.8$}

For $N P 1>0.8$, the eliminated harmonics are expected to reappear due to the non-linearity portion of the trajectory

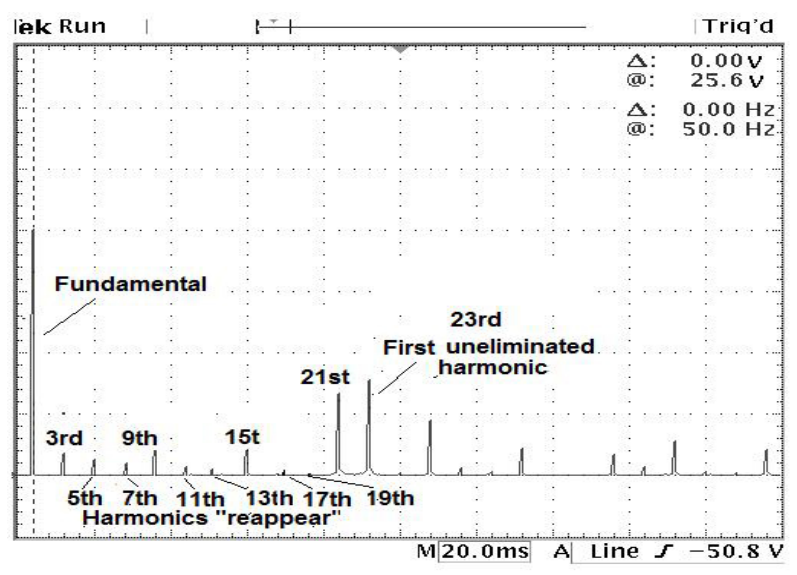

Math

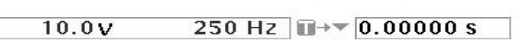

(a)

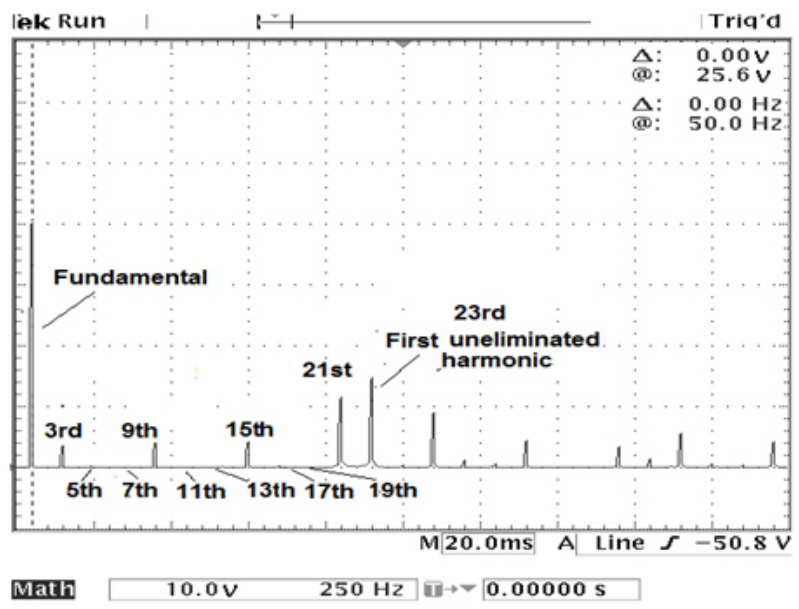

(b)

Fig. 14. Spectra with $m=7, N P 1=1.1$ (a) without correction factor (b) with correction factor. Scale: Amplitude ( $y$-axis): 10V/div. Frequency ( $x$-axis): $250 \mathrm{~Hz} / \mathrm{div}$.

curves. To overcome this, a correction factor is incorporated into the HEPWM switching angle as described by Eqns. (10)(12). Fig. 14 shows the spectra of the output voltage without the correction factor for $m=7$ at $N P 1=1.1$. Careful observation reveals the eliminated harmonics reappear at the 5th, 7th, 9th, 11th, 13th and 17th and 19th. Fig. 14(b) shows the resulting spectra after the correction factor is incorporated. As can be seen, the magnitudes of the harmonics that reappear have been reduced significantly. Figs. 15 and 16 show the performance of the first four eliminated harmonics for values of $m$ up until 17. From these Figs. it can be seen that the elimination performance has been improved between 4 to 6 times. Furthermore, the trend of improved accuracy as $m$ gets higher holds until $m=17$.

\section{CONCLUSIONS}

This paper has proposed an algorithm to calculate the switching angles on-line using a harmonic elimination PWM scheme. The algorithm is an approximation of the trajectories of the exact HEPWM switching angles. It results in generalized polynomial equations which require only the addition and 


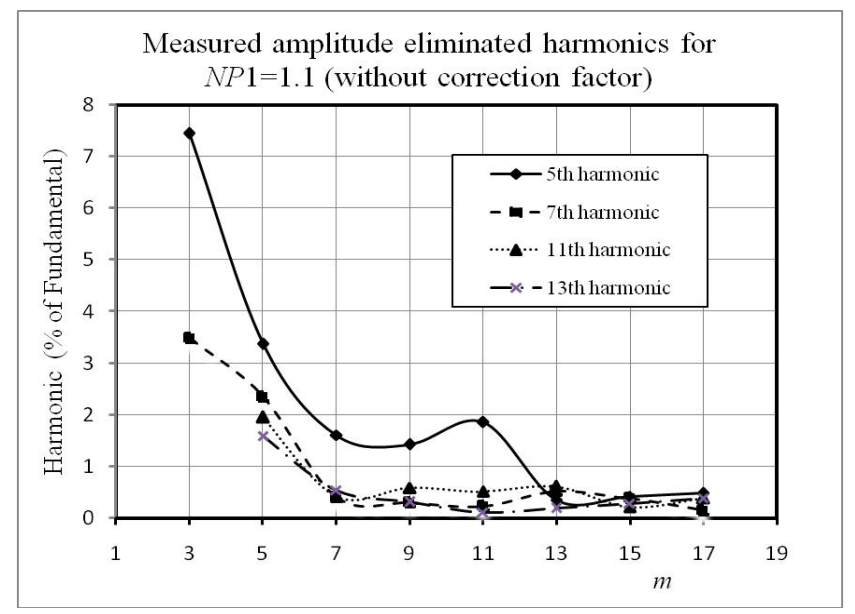

Fig. 15. Performance of the first four eliminated harmonics for different $m$ values at $N P 1=1.1$, without correction factor.

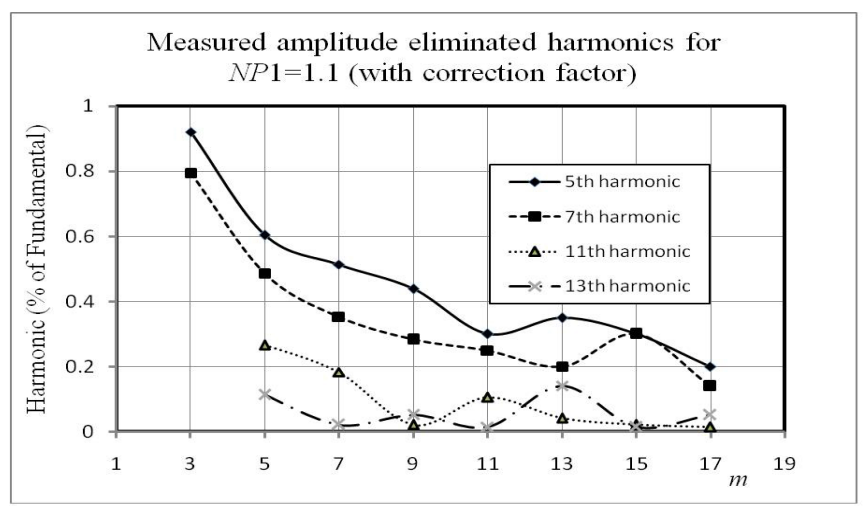

Fig. 16. Performance of the first four eliminated harmonics for different $m$ values at $N P 1=1.1$, incorporating correction factor.

multiplication processes, allowing its implementation using a low cost microprocessor. Changes to the number of harmonics to be eliminated and the fundamental amplitude of the pole switching waveform (NP1) can be calculated independently. This feature is particularly well suited for motor drive applications. An extensive angle error analysis to determine the accuracy of the algorithm is carried out. The maximum difference between the exact angle and the one calculated using this scheme is found to be less than $1^{\circ}$ for $0<N P 1<0.8$. For higher value of $N P 1$, the error is about $8^{\circ}$. However, by incorporating an error correction scheme, the error is reduced by between 3 and 6 times. To verify the workability of the technique, an experimental single phase test VSI was constructed. The algorithm was implemented using a fixed point, 16-bit microprocessor. For comparison, a MATLAB simulation of the same inverter system was carried out. In general, the results obtained from the test rig were in close agreement with the theoretical predictions and simulations. However, it was also observed that for higher switching frequencies $(m$ $>17$ ), the effects of dead time and microprocessor resolution become prominent and should not be overlooked. Further work involving Field Programmable Gate Arrays (FPGA) may be beneficial to extend the workability of the proposed algorithm to higher switching frequencies.

\section{REFERENCES}

[1] Patel H. S. and Hoft, R.G. (1973), "Generalized Techniques of Harmonic Elimination and Voltage Control in Thyristor Inverters: Part I-Harmonic Elimination", IEEE Transaction on Industrial Applications. Vol. IA-9, No.3, pp. 310-317.

[2] Patel H. S. and Hoft, R.G. (1974), "Generalized harmonic elimination and voltage control in thyristor inverters: part II-voltage control technique," IEEE Transaction on Industrial Applications. Vol. IA-10, No. 5, pp. 666-673.

[3] Enjeti, P., and Lindsay, J. F. (1987), "Solving nonlinear equations of harmonic elimination PWM in power control," Electronics Letters, Vol. 23, No. 12, pp. 656-657, Jun. 1987.

[4] Enjeti, P., Ziogas P. D. and Lindsay, J. F. (2000), "Programmed PWM Techniques to Eliminate Harmonics: A Critical Evaluation." IEEE Transaction on Industry Applications. Vol. 26, No.2, pp. 245-253.

[5] Dahidah, M.S.A.; Agelidis, V.G. (2008), "Selective Harmonic Elimination PWM Control for Cascaded Multilevel Voltage Source Converters: A Generalized Formula", IEEE Transactions on Power Electronics, Vol. 23, Issue 4, pp.1620-1630, Jul.

[6] Agelidis, V.G.; Balouktsis, A.I.; Dahidah, M.S.A. (2008), “A Five-Level Symmetrically Defined Selective Harmonic Elimination PWM Strategy: Analysis and Experimental Validation", IEEE Transactions on Power Electronics, Vol. 23, Issue 1, pp.19-26, Jan.

[7] Taufiq, J. A., Mellitt, B. and Goodman, C. J. (1986) "Novel Algorithm for Generating Near Optimal PWM Waveforms for AC Traction Drives" IEEE Proceedings, Vol. 133, Pt. B, No. 2, pp. 85-93.

[8] S. R. Bowes and P. R. Clark (1992) "Simple Microprocessor Implementation of New Regular-Sampled Harmonic Elimination PWM Techniques", IEEE Transaction on Industry Applications, Vol. 28, No.1, pp. 89-95.

[9] Bowes, S.R., Grewal, S. (2000), "Novel space-vector-based harmonic elimination inverter control", IEEE Transactions on Industry Applications, Vol. 36, Issue 2, pp. 549-557, Mar.-Apr.

[10] Agelidis V. G., Balouktsis A., and Balouktsis I. (2004), "On applying a minimization technique to the harmonic elimination PWM control: The bipolar waveform," IEEE Power Electronics Letters, Vol. 2, No. 2, pp. 41-44, Jun.

[11] Liang, T.J., O'Connell, R.M.; Hoft, R.G. (1997), "Inverter harmonic reduction using Walsh function harmonic elimination method", IEEE Trans. on Power Electronics, Vol. 12, Issue 6, Nov., pp.971-982.

[12] Kato, T. (1999), "Sequential homotopy-based computation of multiple solutions for selected harmonic elimination in PWM inverters", IEEE Transactions on Circuits and Systems I: Fundamental Theory and Applications, Vol. 46, Issue 5, pp.586-593, May.

[13] Chiasson, J.N.; Tolbert, L.M.; McKenzie, K.J.; Zhong Du. (2004), "Elimination of harmonics in a multilevel converter using the theory of symmetric polynomials and resultants", IEEE Transactions on Control Systems Technology, Vol. 13, Issue 2, pp. 216-223, Mar.

[14] Zhong D; Tolbert, L.M.; Chiasson, J.N. (2006), "Active harmonic elimination for multilevel converters", IEEE Transactions on Power Electronics, Vol. 21, Issue 2, pp.459-469, Mar.

[15] Tolbert, L.M.; Chiasson, J.N.; Zhong Du; McKenzie, K.J. (2005), "Elimination of harmonics in a multilevel converter with nonequal DC sources", IEEE Transactions on Industry Applications, Vol. 41, Issue 1, pp. 75-82, Jan.-Feb.

[16] Ozpineci, B.; Tolbert, L.M.; Chiasson, J.N. (2005), "Harmonic optimization of multilevel converters using genetic algorithms", IEEE Power Electronics Letters, Vol. 3, Issue 3, pp.92-95, Sep.

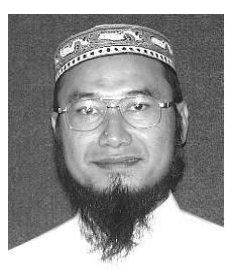

Zainal Salam obtained his B.Sc., M.E.E. and Ph.D. from the University of California, Universiti Teknologi Malaysia (UTM) and the University of Birmingham, UK, in 1985, 1989 and 1997, respectively. He has been a lecturer at UTM for 24 years and is now a Professor in Power Electronics with the Faculty of Electrical Engineering. He has been working on several research projects and consulting jobs on battery powered converters. Currently he is the Director of the Inverter Quality Control Center (IQCC) UTM which is responsible for testing PV inverters that are to be connected to the local utility grid. His research interests include all areas of power electronics, renewable energy, power electronics and machine control. 